ARTICLE

Received 1 Jul 2014 | Accepted 2 Oct 2014 | Published 20 Nov 2014

DOI: $10.1038 /$ ncomms 6461

\title{
Giant enhancement in vertical conductivity of stacked CVD graphene sheets by self-assembled molecular layers
}

Yanpeng Liu ${ }^{1,2, \star}$, Li Yuan ${ }^{1, \star}$, Ming Yang ${ }^{3}$, Yi Zheng ${ }^{1}$, Linjun Li ${ }^{1}$, Libo Gao ${ }^{1}$, Nisachol Nerngchamnong ${ }^{1}$, Chang Tai Nai ${ }^{1,4}$, C.S. Suchand Sangeeth ${ }^{1}$, Yuan Ping Feng ${ }^{3}$, Christian A. Nijhuis ${ }^{1} \&$ Kian Ping Loh $^{1}$

Layer-by-layer-stacked chemical vapour deposition (CVD) graphene films find applications as transparent and conductive electrodes in solar cells, organic light-emitting diodes and touch panels. Common to lamellar-type systems with anisotropic electron delocalization, the planeto-plane (vertical) conductivity in such systems is several orders lower than its in-plane conductivity. The poor electronic coupling between the planes is due to the presence of transfer process organic residues and trapped air pocket in wrinkles. Here we show the plane-to-plane tunnelling conductivity of stacked CVD graphene layers can be improved significantly by inserting 1-pyrenebutyric acid $N$-hydroxysuccinimide ester between the graphene layers. The six orders of magnitude increase in plane-to-plane conductivity is due to hole doping, orbital hybridization, planarization and the exclusion of polymer residues. Our results highlight the importance of interfacial modification for enhancing the performance of LBL-stacked CVD graphene films, which should be applicable to other types of stacked two-dimensional films.

\footnotetext{
${ }^{1}$ Department of Chemistry, Graphene Research Center, National University of Singapore, 3 Science Drive 3117543 Singapore, Singapore. ${ }^{2}$ NanoCore, National University of Singapore, 117576 Singapore, Singapore. ${ }^{3}$ Department of Physics, National University of Singapore, 2 Science Drive 3, 117551 Singapore, Singapore. ${ }^{4}$ NUS Graduate School for Integrative Sciences and Engineering, 28 Medical Drive \#05-01, 117597 Singapore, Singapore. * These authors contributed equally to this work. Correspondence and requests for materials should be addressed to C.A.N. (email: christian.nijhuis@nus.edu.sg) or to K.P.L. (email: chmlohkp@nus.edu.sg).
} 
arge-area graphene produced by chemical vapour deposition (CVD) is an attractive candidate for transparent and conducting electrodes due to its transparency, high electrical conductivity and flexibility. CVD graphene has been deployed as diffusion barriers in electrode modifiers, transparent conductors in touch screen panels, organic light-emitting diodes and solar cells ${ }^{1-7}$. However, the conductivity of pristine graphene is limited by the amount of carriers, and further modification such as chemical or electrostatic doping is needed to improve the conductivity. One way to improve the conductivity is to stack single-layer CVD graphene in a layer-by-layer (LBL) manner to form multilayers $2,4,5,7$. Although the overall conductivity (inplane or out-of-plane) improves with layer thickness, weak electronic coupling between the layers prevents a linear scaling of the conductivity with thickness of the stacked layers. These problems will be amplified in large-area electronic devices where series resistance scales with lateral dimension. Most of the research efforts on graphene have thus far focused on improving the in-plane lateral conductivity, while scant attention has been paid to the plane-to-plane (vertical) conductivity, which is important in electrode applications. There is a need to probe the quality of interfaces in stacked CVD graphene films and to correlate that with the electronic properties. Pyrene and its derivatives are widely used to functionalize graphene sheets due to their $\pi-\pi$ stacking ability, they can also act as hole-transport materials in photovoltaic devices due to their strong electron delocalization $^{8-10}$.

In conventional LBL wet transfer process, the vertical conductivity of stacked CVD graphene films is significantly lower than that of graphite or dry-stacked mechanically exfoliated graphene films ${ }^{11}$. The reasons for low conductivity in LBLstacked graphene films include the following: first, stacked graphene films trap solvent and moisture, hence the measured thickness of monolayer graphene using atomic force microscopy (AFM) is $\sim 0.7-1.0 \mathrm{~nm}$ compared with the $0.34 \mathrm{~nm}$ of exfoliated graphene ${ }^{4,12,13}$; second, wrinkles on the films arising from surface tension during wet transfer trap air pockets and prevent good electronic coupling between the graphene layers ${ }^{3,4,14}$; and third, polymer residues from the transfer process ${ }^{14-17}$. These factors not only introduce scattering centres to in-plane carrier transport, but also contribute to weak interlayer electronic coupling, as depicted in Fig. 1a.

Here we study whether the electronic coupling of stacked CVD graphene layers can be improved by inserting self-assembled molecules (SAM) between the layers, as measured via the EGaIn (eutectic gallium indium) technique. Through the insertion of a self-assembled monolayer (1-pyrenebutyric acid $N$-hydroxysuccinimide ester, PBASE) between two LBL-stacked graphene films, we find that the interfacial resistance can be mitigated to a large extent (current density: pristine one $\sim 10^{-3} \mathrm{~A} \mathrm{~cm}^{-2}$; modified one $\sim 10^{3} \mathrm{~A} \mathrm{~cm}^{-2}$ ). The large pendant $N$-hydroxysuccinimide ester group of PBASE blocks a second layer of molecules from stacking on top. This self-limiting nature of PBASE in forming a monolayer is important, as insertion of thick dielectric layers would weaken the interlayer coupling. Other small molecules with similar pyrene backbone structures, but with pendant groups that can undergo intermolecular bonding, are prone to aggregation (1-pyrenecarboxylic acid, 1-pyrenebutanol, like hydrogen bonding), which produces deleterious effects on the conductivity $^{18}$. Our study highlights the importance of selecting the molecules with the right chemical structure as an insertion layer to enhance the electrical performance of LBL-stacked CVD graphene films.

\section{Results}

Plane-to-plane conductivity measurement using EGaIn. To study the plane-to-plane conductivity of stacked CVD graphene films, we used a non-invasive liquid metal alloy top electrode (EGaIn) to contact the upper graphene films softly ${ }^{19,20}$. This method yields junctions in high yields (up to 100\%) with good stability because of the presence of a thin $(\sim 0.7 \mathrm{~nm})$ highly conductive layer of $\mathrm{Ga}_{2} \mathrm{O}_{3}$ that forms spontaneously in air ${ }^{21}$. This protective layer of conductive oxide prevents the bulk metal from diffusing into the structures of interest and adds to the mechanical stability of the junction ${ }^{22}$. This method also makes
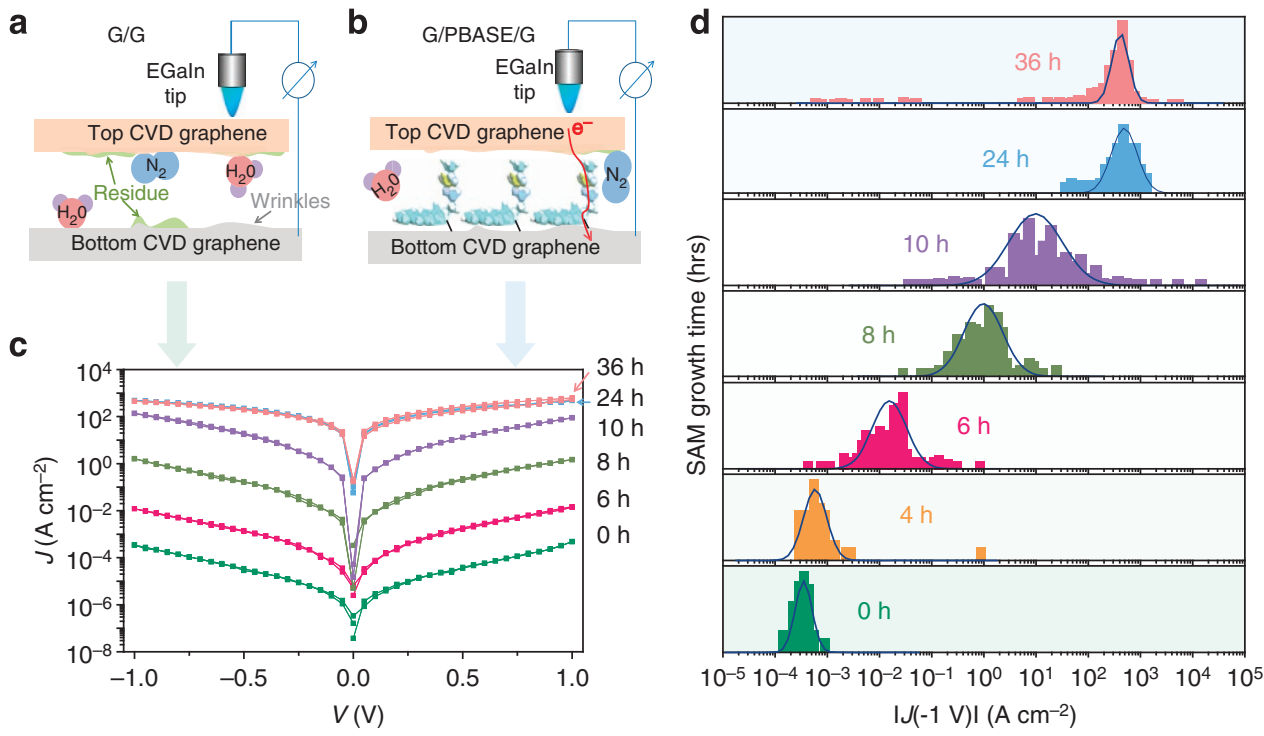

Figure 1 | Schematic illustrations of EGaln measurements and the time evolution of current density. (a,b) Schematic drawings of the system of recording the vertical tunnelling current density across two LBL-stacked CVD graphene films. Under ideal conditions, the gap between top and bottom graphene films determines the tunnelling resistance and organic residues reduce interlayer coupling in model (a), which is the case for stacked CVD graphene. (c) Experimental data of current density as a function of SAM growth time. The log-standard error bars are omitted for visual comparison purpose. The full version could be found in Supplementary Fig. 3. (d) Histograms of the absolute tunnelling current density $(J)$ distributions with various PBASE SAM growth times at a bias of $-1 \mathrm{~V}$. The Gaussian fitting curves are represented in blue. 
a

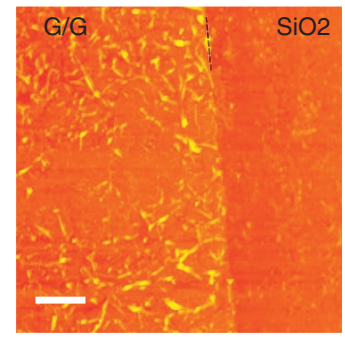

c

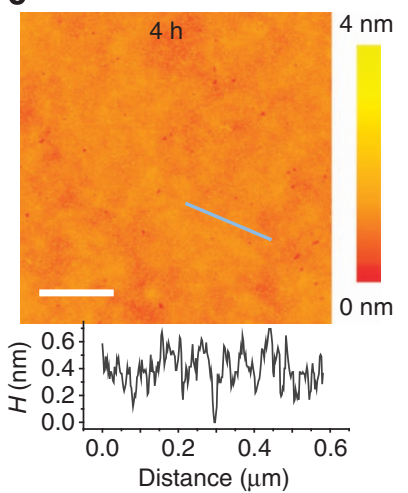

e
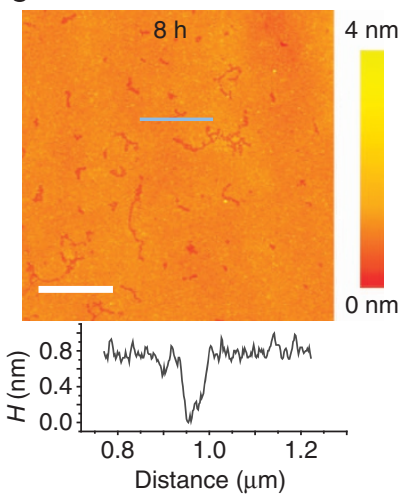

b

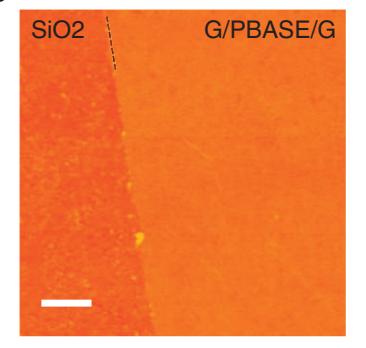

d

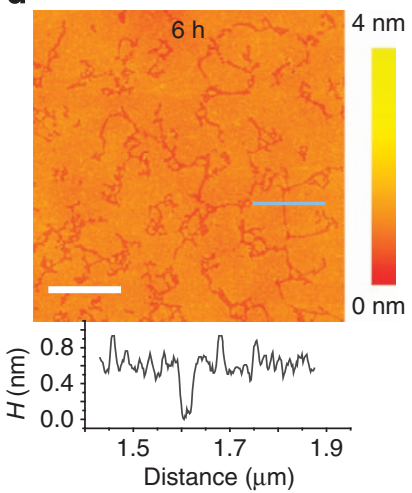

f

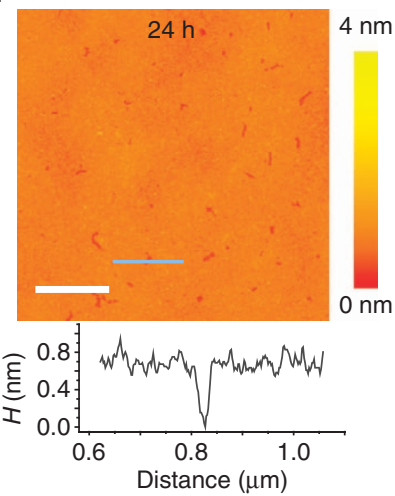

Figure 2 | AFM imaging and representative height profiles.

$(\mathbf{a}, \mathbf{b})$ Topographies of two LBL-stacked CVD graphene before (G/G) and after (G/PBASE/G) the insertion of PBASE. The corrugations on G/PBASE/G films are obviously smaller than $G / G$ films. The thickness of this bilayer CVD graphene is $\sim 0.7-1.0 \mathrm{~nm}$. Scale bar, $1 \mu \mathrm{m}$. (c-f) Time sequence of PBASE self-assembly on CVD graphene (PBASE/G). Scale bar, $500 \mathrm{~nm}$. Topography line scans show that the height of PBASE SAM layer is $\sim 0.7 \pm 0.1 \mathrm{~nm}$.

it possible to fabricate large numbers of highly repeatable junctions for collecting statistics ${ }^{19-21}$. In contrast, conventional methods require harsh fabrication methods (for example, direct metal deposition of large electrode on graphene) and sometimes cause damage (mainly penetration) to the graphene layers resulting in short or junctions dominated by artefacts 22 .

High-quality graphene films grown by CVD were transferred onto ultra-flat gold substrate $(\mathrm{Cr} / \mathrm{Au}, 5 / 100 \mathrm{~nm}$ as bottom electrode deposited at very low deposition rate) via conventional PMMA(Poly(methyl methacrylate))-assisted wet transfer process. After removing PMMA layer, the samples were immersed in $5 \mathrm{mM}$ PBASE in dimethylformaldehyde (DMF) solution at $80^{\circ} \mathrm{C}$ to allow the PBASE molecules to form a SAM layer on graphene via $\pi-\pi$ interactions ${ }^{23}$. After drying, another CVD-grown graphene layer was directly stacked onto to form graphene/ PBASE/graphene sandwich architecture (schematic drawing in

\begin{tabular}{|c|c|c|c|c|}
\hline $\begin{array}{l}\text { Growth } \\
\text { time } \\
\end{array}$ & $\begin{array}{c}\text { Number of } \\
\text { junctions }\end{array}$ & $\begin{array}{c}\text { Number of } \\
\text { shorts }\end{array}$ & $\begin{array}{c}\text { Number of } \\
\text { traces }\end{array}$ & $\begin{array}{c}\text { Yield of } \\
\text { junctions (\%) }\end{array}$ \\
\hline 0 & 15 & 2 & 360 & 86.7 \\
\hline 4 & 15 & 1 & 360 & 93.3 \\
\hline 6 & 15 & 1 & 360 & 93.3 \\
\hline 8 & 15 & 2 & 360 & 86.7 \\
\hline 10 & 15 & 1 & 360 & 93.3 \\
\hline 24 & 15 & 0 & 360 & 100 \\
\hline 36 & 15 & 0 & 360 & 100 \\
\hline
\end{tabular}

Fig. $1 b$ and typical AFM images of G/PBASE/G in Fig. 2b). Ripples induced by surface tension, cracks and polymer particle residues from wet transfer process are usually found on the surface of as-growth graphene films, as shown in Fig. 2a. After the assembly of PBASE, most of these defects vanish, except for some pin holes arising from the incomplete coverage of PBASE. Due to strong $\pi-\pi$ stacking on graphene, the self-assembly of PBASE is able to supplant polymer residues (see Supplementary Fig. 1 for dark-field images illustrating the exclusion of polymer residues by PBASE monolayer growth) and also planarizes the defective graphene surface. The binding of a strong layer of PBASE increases the bending rigidity and reduces the rippling (smooth surface in Fig. 2c-f; similar phenomenon occurs on thick graphene films in Supplementary Fig. 2 and Supplementary Note 1). In addition, the hydrophilic side chain improves the wetting properties of graphene and avoids ripples formation during wet transfer process of top graphene layer. All these factors contribute to strong interlayer coupling between the top and bottom CVD graphene layers.

Using the EGaIn technique, large amount of statistical current density versus voltage $(J(V))$ data was readily collected (For more information, please find Supplementary Note 2). Table 1 shows the $J(\mathrm{~V})$ data for SAMs formed over time period ranging from 4 to $36 \mathrm{~h}$. For each device with specified SAM growth time, 360 traces were recorded for three batches of samples to calculate $\log |J|^{19-21}$. The corresponding AFM images (in Fig. 2c-f) show that these SAMs saturate at a thickness of $0.7 \pm 0.1 \mathrm{~nm}$ due to its self-terminating ability. This is different from the self-assembly behaviour of aromatic organic molecules where longer assembly time leads to multilayer formation ${ }^{24}$. As can be seen in Fig. 1c, the log-average current density $(J)$ increases sharply to $10^{3} \mathrm{~A} \mathrm{~cm}^{-2}$ and saturates after $24 \mathrm{~h}$ of SAM growth (for $J(V)$ curves, see Supplementary Fig. 3). The tunnelling current density $J$ (in $\mathrm{A} \mathrm{cm}^{-2}$ ) can be described by the simplified form of the Simmons equation

$$
J=J_{0} e^{-\beta d}
$$

where $J_{0}\left(\mathrm{~A} \mathrm{~cm}^{-2}\right)$ is the current density for junction with $d=0 \AA$ and $\beta\left(\AA^{-1}\right)$ is the tunnelling decay coefficient ${ }^{19-21,25}$. The relative low current density in the first $4 \mathrm{~h}$ of SAM growth probably comes from the induction time needed for nucleation and organization of the SAM (Fig. 2c). Figure 1d presents statistical distribution histograms of the log-average $J$ curves at a bias of $-1 \mathrm{~V}$, where the data is collected as a function of growth time of the SAM. The good Gaussian-fitted histograms illustrate that stable and uniform junctions are formed over large area of the sample. As shown in Fig. 1d, the current density increases from $\sim 10^{-3} \mathrm{~A} \mathrm{~cm}^{-2}$ of stacked $\mathrm{G}-\mathrm{G}$ layers to $\sim 10^{3} \mathrm{~A} \mathrm{~cm}^{-2}$ of G-PBASE-G after the formation of a full monolayer of PBASE, which is a six order enhancement in vertical conductivity. Statistics have been collected from 105 

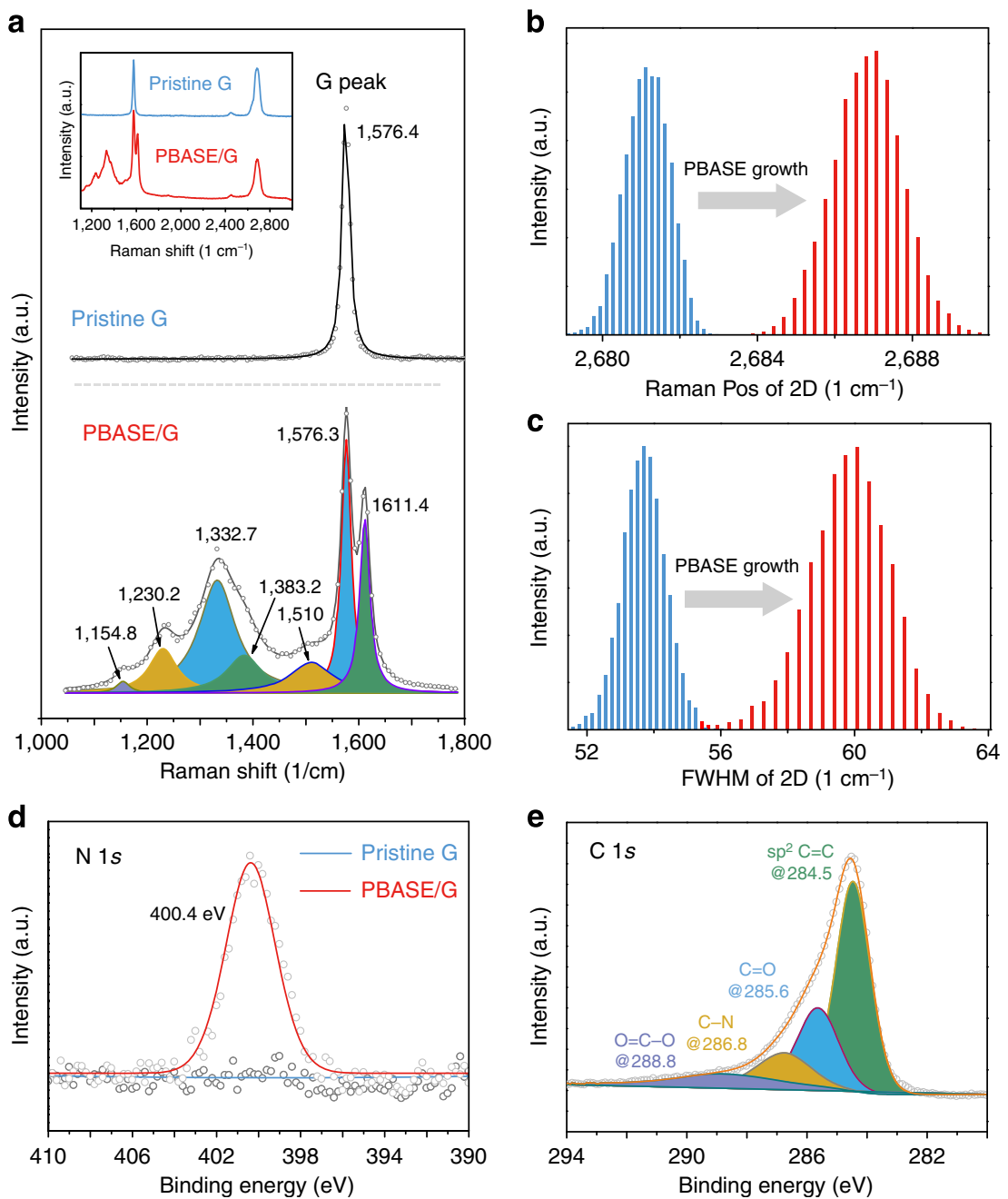

Figure 3 | Raman and XPS studies of CVD graphene films adsorbed with a PBASE monolayer. (a) Representative Raman spectra before and after PBASE SAM growth at the same spot near the $G$ band. The inset figure of a shows the overall Raman spectrums comparison. (b,c) Histograms showing the position and FWHM extracted from Raman colour mapping over the same area. (d,e) High-resolution N 1s and C 1s XPS spectrum of CVD graphene adsorbed with PBASE, respectively. XPS, X-ray photoelectron spectroscopy.

regions of our samples from three batches $(2,520$ traces in total), which shows consistently five to six order enhancement (statistics histogram, see Supplementary Fig. 3). It is worth noting also that the in-plane conductivity of two stacked CVD graphene films is doubled after the introduction of PBASE layer (see Supplementary Fig. 4 and Supplementary Note 3). The enhancement along in-plane direction is much smaller than that out-of-plane direction, due to different transport mechanism $^{11}$.

Characterizing PBASE-adsorbed CVD graphene. Raman spectroscopy was employed to study the graphene film after its interaction with $\mathrm{SAM}^{26-28}$. A spatial colour map of the bottom graphene using $532 \mathrm{~nm}$ excitation laser was carried out before and after SAM growth over the same area (see Supplementary Fig. 5). In the low-frequency regions as shown in Fig. 3a, the doping dependence of the $G$ band is not as sensitive as the two-dimensional (2D) band, but several intrinsic signals due to PBASE molecules appear. The peak at $1,332.7 \mathrm{~cm}^{-1}$ arises from $\mathrm{sp}^{3}$ bonding. The peak at $1,611.4 \mathrm{~cm}^{-1}$ is assignable to pyrene group resonance and the peak at $1,383.2 \mathrm{~cm}^{-1}$ is due to the introduction of disorder arising from orbital hybridization of the molecule with graphene plane. The mapping images of $2 \mathrm{D}$ peaks are transformed to histograms for visual comparison. After SAM growth, the $2 \mathrm{D}$ peak of the graphene films is shifted to higher frequency $\left(\sim 6 \mathrm{~cm}^{-1}\right.$; Fig. $\left.3 \mathrm{~b}\right)$ and has wider FWHM (full-width at half-maximum; $\sim 6 \mathrm{~cm}^{-1}$; Fig. $3 \mathrm{c}$ ), which is a signature of hole doping by $\mathrm{PBASE}^{26,28}$. Ultraviolet photoelectron spectroscopy was used to record the work function change induced by the adsorption of PBASE. Consistent with hole doping, the work function is increased by $\sim 0.3 \mathrm{eV}$ as shown in Supplementary Fig. 6a (refs 28,29). Back-gate field-effect transistor was also fabricated to investigate the doping effect of PBASE molecules (see Supplementary Fig. 7 and Supplementary Note 4). The results show that the doping level of CVD graphene films by PBASE molecules could be up to $\sim 4 \times 10^{12} \mathrm{~cm}^{-2}$ (the intrinsic carrier density of pristine CVD bilayer graphene is in the order of $10^{11} \mathrm{~cm}^{-2}$ ). The enhanced carrier density will certainly improve the overall conductivity (for in-plane conductivity, see Supplementary Fig. 4), although the magnitude of increase observed for the vertical conductivity far exceeds contribution due to increased carrier density alone.

The quality of the self-assembly monolayer is analysed by $\mathrm{X}$-ray photoelectron spectroscopy. The wide scans show that no metallic element, arising from catalyst or etchant, is detected (see Supplementary Fig. 8a). The presence of PBASE is confirmed by the characteristic N $1 s$ peak at $400.4 \mathrm{eV}$ (Fig. 3d). The C $1 s$ peak 

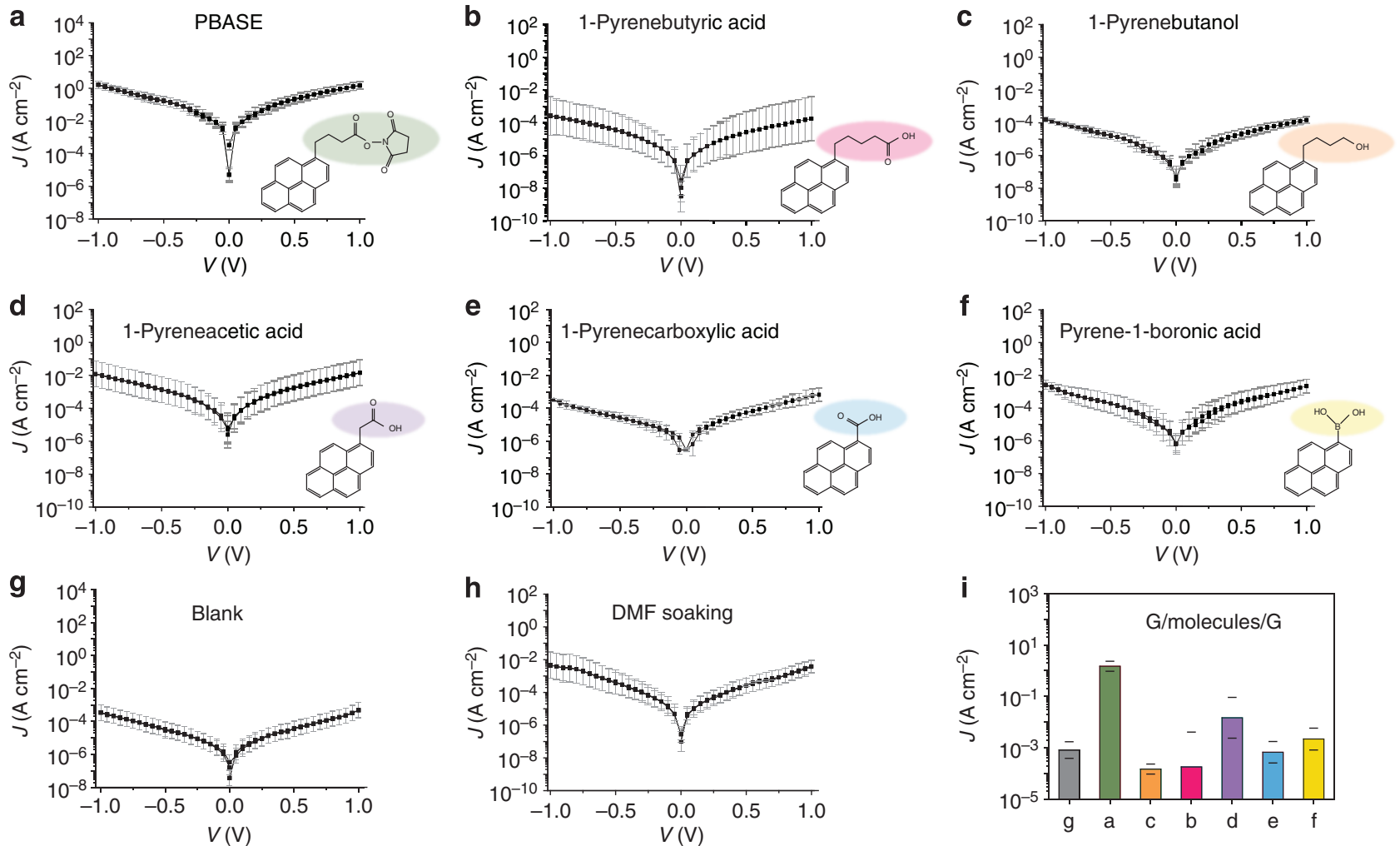

Figure 4 | Comparing the current density in G-SAM-G sandwich structure with different SAMs. (a-f) $J(V)$ curves of G/PBASE/G, G/1-pyrenebutyric acid/G, G/1-pyrenebutanol/G, G/1-pyreneacetic acid/G, G/1-pyrenecarboxylic acid/G and G/pyrene-1-boronic acid/G devices, respectively. (g) J(V) curves of directly LBL-stacked $G / G$ devices. (h) $J(V)$ curves of LBL-stacked $G / G$ devices with bottom graphene soaking in DMF solvent for 8 h.

(i) The average $J$ characteristics of $G /$ molecules/G device with a bias of $1.0 \mathrm{~V}$ for all these devices. All SAMs were grown for $8 \mathrm{~h}$.

can be deconvoluted into a weak $\mathrm{C}-\mathrm{N}$ peak at $286.8 \mathrm{eV}$ and the main $\mathrm{C} 1 \mathrm{~s}$ peak at $284.5 \mathrm{eV}$, as shown in Fig. 3e. The asymmetric stretching modes of PBASE are detected by Fourier transform infrared spectroscopy at $3,040,2,935$ and $2,868 \mathrm{~cm}^{-1}$ for alkyl group stretching and one sharp peak at $848 \mathrm{~cm}^{-1}$ from C-H wagging vibration (see Supplementary Fig. 6b. For more characterizations, see Supplementary Figs 12-16).

Conductivity enhancement by PBASE for stacked CVD graphene. To investigate the relationship between molecular structure and the ability of the molecules to act as effective molecular linkers, six pyrene derivatives with different pendant groups were self-assembled on CVD graphene. Several hundred data were collected for each G/molecules/G sandwich device (for statistics histogram, see Supplementary Fig. 9). It is obvious that G/PBASE/G forms the most stable junction devices and has a good uniformity. To get rid of conductivity contribution from the solvent, a control sample was soaked in pure DMF solvent under the same condition and very marginal change is observed. The log-average $J$ values at a bias of $1 \mathrm{~V}$ with log-s.d. error bar are summarized in Fig. $4 \mathrm{i}$ for comparison purposes. It was found that molecules that can undergo intermolecular hydrogen bonding tend to aggregate and result in poor conductivity when used as molecular linkers. The bond energy of $\pi-\pi$ stacking is typically $<10 \mathrm{kcal} \mathrm{mol}^{-1}$, which is comparable to moderate and weak hydrogen bond energy. Hence, for pyrene derivatives which can undergo intermolecular hydrogen bonding, its packing order and structure will be determined by competition between $\pi-\pi$ stacking and hydrogen bonding. The topography of the SAM formed by two molecules, 1-pyrenecarboxylic acid and 1-pyrenebutanol on graphene, illustrates these competing effects. For 1-pyrenebutanol, the SAM suffers from some degree of aggregation (see Supplementary Fig. 10 and Supplementary Note 5). The presence of molecular clusters increases the tunnelling distance and blocks the charge transfer, resulting in poor current density. Moreover, our density functional theory (DFT) calculation shows that unlike PBASE, 1-pyrenebutanol does not undergo effective orbital hybridization with graphene (see below 'DFT simulation' section). In the case of 1-pyrenecarboxylic acid, the thickness of molecular layer is $\sim 2 \mathrm{~nm}$. The structure can be described by two hydrogen-bonded repeating units in the herringbone structure (see Supplementary Fig. 10e,f), originating from the strong intermolecular hydrogen bonding of the $\mathrm{COOH}$ groups ${ }^{18}$. In this case, the increase in interplanar distance $d$ lowers the conductivity significantly (equation (1)) and offsets any positive effects of orbital hybridization of 1-pyrenecarboxylic acid with graphene. Although PBASE molecules have the largest side chain group among these molecules, its large pendant $N$-hydroxysuccinimide ester group blocks a second layer of molecules from stacking on top, hence it is self-limiting at a monolayer, thus ensuring that the tunnelling distance does not exceed the thickness of the monolayer. Stacked CVD graphene layers typically have an interplanar distance that is much larger than the van der Waals distance due to inclusions of polymer residues and wrinkles, substitution of these by PBASE narrows the physical gap and results in enhancement of the tunnelling current.

Influence of PBASE on mechanically exfoliated graphene stack. In the case of mechanically exfoliated graphene flakes, which are stacked by the dry transfer method, the coupling between the stacked graphene layers is better than that of CVD graphene due to the absence of polymer residues and wrinkles ${ }^{30-32}$. In this case, the interplanar separation is close to the van der Waals distance and the insertion of PBASE between the layers actually widens the 

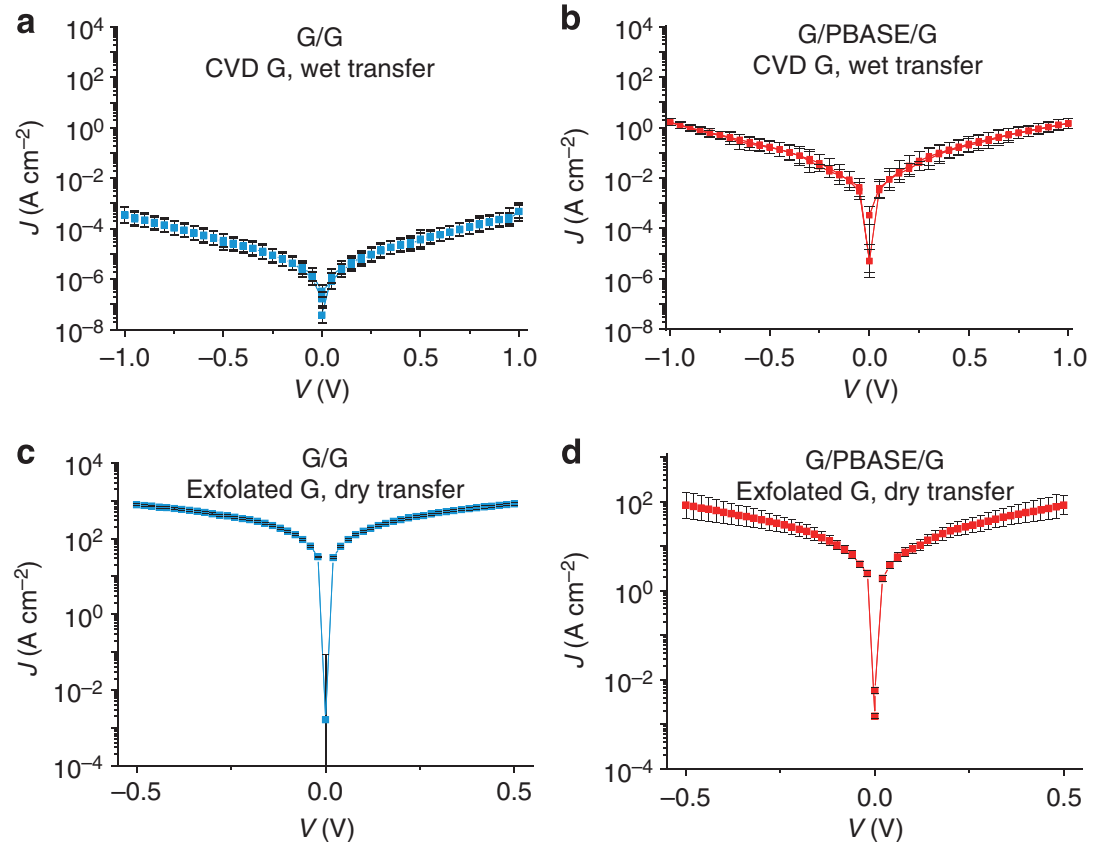

Figure 5 | Average $J(V)$ curves' comparison of devices prepared by different methods. (a,b) $J(V)$ curves of G/G and G/PBASE/G devices via wet transfer of CVD graphene. (c,d) $J(V)$ curves of $G / G$ and $G / P B A S E / G$ devices via dry transfer of micromechanically cleaved graphene monolayer. The error bars refer to the log-s.d. of the log-average $J$ values.

spacing, and will be expected to reduce the tunnelling current. To verify if this is true, the $I-V$ curves of devices made from mechanically exfoliated graphene flakes that are stacked by dry transfer method are recorded with four-probe stations and listed in Fig. 5c, where we compare the tunnelling current density of $\mathrm{G} / \mathrm{G}$ and G/PBASE/G. In contrast to the conductivity enhancement for stacked CVD graphene system reported earlier (Fig. 5a,b), there is now a slight reduction in the current from $\sim 8.0 \times 10^{2}$ (linear $I-V$ curve, at $|0.5 \mathrm{~V}|$ bias) to $8.2 \times 10^{1} \mathrm{~A} \mathrm{~cm}^{-2}$ after the introduction of PBASE SAM layer between two graphene flakes. From equation (1), the value of $\beta$ will vary under different junction systems. In graphene-insulator tunnelling system, the tunnelling current density has an exponential dependence on the thickness of the tunnelling barrier $d$. Compared with graphene-boron nitride-graphene system, with tunnel distance of $\Delta d \approx 6 \AA$, there should be a three order of magnitude drop in current density from graphenegraphene device ${ }^{33}$. However, as shown in Fig. $5 c$,d, the current density only decreases by one order of magnitude, which reveals that the presence of SAM molecules as an interlayer has a positive effect in enhancing the vertical conductivity to counteract the current decay due to the increase in tunnelling distance, although the effect of the latter, which has an exponential dependence, dominates the current density eventually. We can infer that compared with mechanically exfoliated, dry-transferred graphene, the much larger increase in tunnelling current density in the case of stacked CVD graphene after inserting PBASE is due to the supplanting of polymer residues as well as suppression of wrinkles.

DFT calculations of structure and electronic properties. To understand why subtle changes in the functional groups of the pyrene-based SAM affects the vertical conductivity when it is inserted between two graphene layers, DFT calculations were performed to investigate effect of PBASE molecules on electronic properties of two stacked CVD graphene. First, the out-of-plane separation $(d)$ between two graphene sheets illustrated in Fig. 6a (left) was adjusted to investigate the separation-dependent tunnelling barrier and out-of-plane band gap, where the tunnelling barrier $(\Delta V)$ is defined as the potential energy above the associated Fermi energy ${ }^{29,34-36}$. As expected, both the tunnelling barrier and out-of-plane band gap of two graphene sheets are almost constant with $d$ varying from 14 to $8 \AA$ and then decrease dramatically with further reduction of out-of-plane separation $d$. The interfacial configuration of PBASE molecular layers shown in Fig. 6c (left) is most energetically favourable, in which the calculated separation between top and bottom graphene is about $13.4 \AA$, in good agreement with above experimental result. The equilibrium distance between molecules and bottom graphene layer $\left(d_{\mathrm{eq}}^{b}\right)$ is $2.3 \AA$ (Fig. 6c) and is similar to typical metalgraphene chemisorption separation distance ( $\mathrm{Pd}, d_{\mathrm{eq}}=2.3 \AA$ ). From the partial density of states (see Supplementary Fig. 11a), the $p$ orbital of $\mathrm{C}$ atom at bottom graphene layer and $s$ orbital of nearest $\mathrm{H}$ atom in PBASE molecule are slightly hybridized ${ }^{29}$. With PBASE as the molecular linker, the tunnelling barrier decreases by $0.98 \mathrm{eV}$ (from 4.22 to $3.24 \mathrm{eV}$ ). It is noted that the energy gap for pure two graphene layers at this separation distance is about $4.06 \mathrm{eV}$ at $M$-point. After the introduction of SAM in between graphene layers, the out-of-plane band gap appears at $\kappa$-point and is reduced to $0.71 \mathrm{eV}$. Therefore, the combined effects of orbital hybridization, reduction in band gap (by $3.35 \mathrm{eV}$ ) and tunnelling barrier contribute to the significant enhancement of vertical conductivity after SAM insertion. In addition, from the out-of-plane band structure in Fig. 6d, the shifting of the Fermi level towards lowest unoccupied molecular orbital also indicates that holes are donated by the absorbed PBASE molecules to graphene, resulting in p-doping. However, for the G/1-pyrenebutanol/G fully relaxed structure, $d_{\mathrm{eq}}^{b}$ and $d_{\mathrm{eq}}^{t}$ ( $d_{\text {eq }}^{t}$, the most energetically favourable distance between molecules and the top graphene) increase to 2.5 and $3.6 \AA$, respectively, as shown in Fig. 6e, which increase the tunnelling barrier height $\Delta V$ to $3.41 \mathrm{eV}$ (3.24 eV for PBASE molecules). Although its out-of-plane band gap is also at $\kappa$-point, the reduction in energy gap $(3.09 \mathrm{eV}$, from $\sim 4.06$ to $0.97 \mathrm{eV})$ is smaller than that of PBASE molecules. Beside this, the orbital 
a
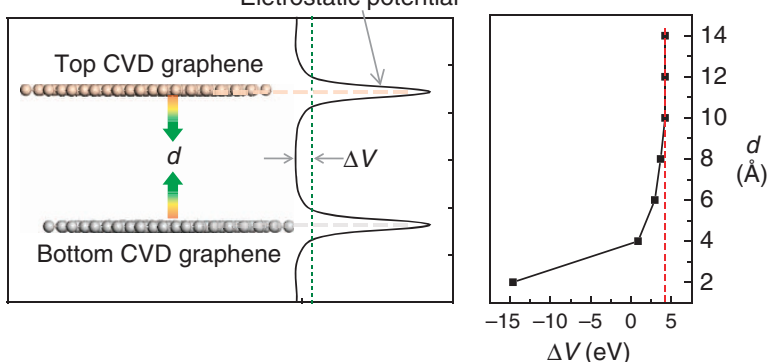

C

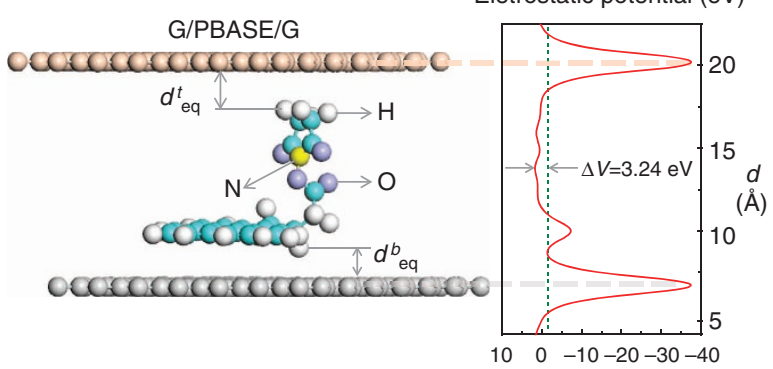

e

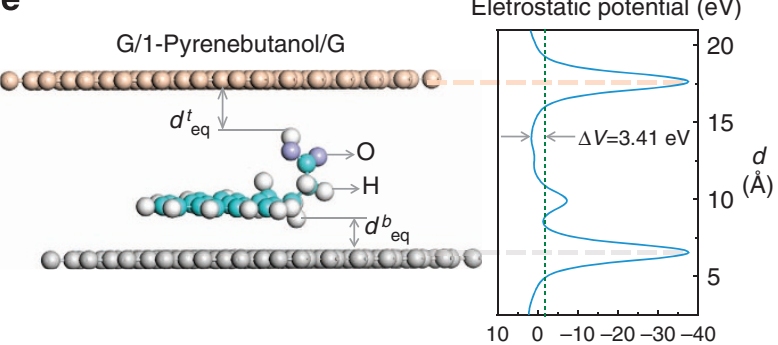

b

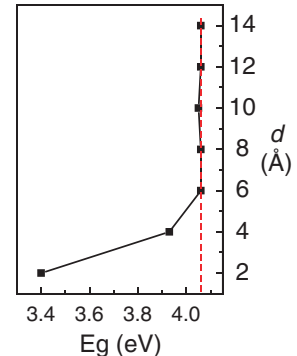

d

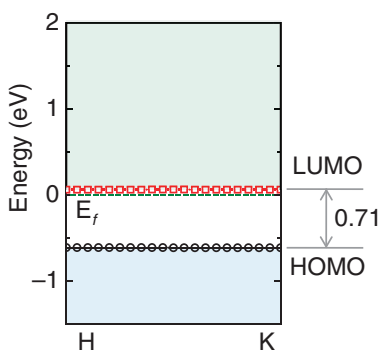

f

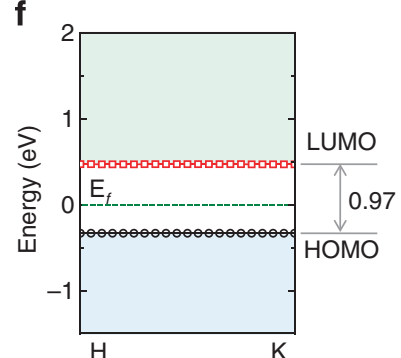

Figure 6 | DFT-simulated structures and their electronic properties. (a) Typical electrostatic potential plot and tunnelling barrier height $\Delta V$ as a function of separation distance between two graphene layers $(d)$. (b) $d$-dependent band gap. (c) Left, optimized G/PBASE/G structure, where $d_{\mathrm{eq}}^{t}=2.9 \mathrm{~A}$ and $d_{\mathrm{eq}}^{b}=2.3 \mathrm{~A}$; right, its corresponding electrostatic potential. (d) Band structure of G/PBASE/G at $\kappa$-point. (e) Left, optimized G/1-pyrenebutanol/G device. The $d_{\mathrm{eq}}^{t}$ and $d_{\mathrm{eq}}^{b}$ are about 3.6 and $2.5 \AA$; right, its corresponding electrostatic potential. (f) Band structure of G/1-pyrenebutanol/G at $\kappa$-point.

hybridization of 1-pyrenenbutanol with graphene is much weaker (see Supplementary Fig. 11b). These results illustrate that PBASE molecules are unique in bridging two stacked CVD graphene sheets and improving its out-of-plane conductivity.

\section{Discussion}

Our studies reveal that the poor plane-to-plane charge transport of LBL-stacked CVD graphene films, which is related to organic inclusions in the interlayers and wrinkles inherited from the wet transfer process, can be alleviated by using self-assembled PBASE as a molecular linker between the stacked graphene sheets. With PBASE inserted between two stacked CVD graphene, the planeto-plane conductivity is enhanced by six orders of magnitude (current density increases from $\sim 10^{-3}$ to $\sim 10^{3} \mathrm{~A} \mathrm{~cm}^{-2}$ ). The improvement is due to a combination of factors, which include doping, planarization effect of PBASE on wrinkles created during the growth or transfer process, as well as the supplanting of polymeric residues by PBASE, which undergoes strong $\pi-\pi$ stacking with graphene. The unique molecular structure of PBASE with a pyrene base and a pendant side group also favours orbital hybridization and molecular bridging between the graphene layers. The conductivity enhancement by molecular linkers applies mainly to wet-transferred film. On dry-transferred, mechanically exfoliated graphene film, the presence of PBASE contributes to a one order reduction in vertical conductivity. Nevertheless, the use of self-assembled molecular layers as molecular plaster to improve the electronic coupling between two stacked layers remains highly relevant to large area, CVDgrown 2D films, since these are generally transferred by wet transfer methods, and are thus affected by wrinkles and organic inclusions when stacked in LBL manner.

\section{Methods}

Junction of CVD graphene via wet transfer. Using PMMA as a backing layer similar to the transfer methods reported in the previous literatures, the as-grown films were transferred onto $\mathrm{Au} / \mathrm{Cr}(100 \mathrm{~nm} / 5 \mathrm{~nm})$ substrates ${ }^{3,5}$. The graphene films were soaked in $5 \mathrm{mM}$ PBASE in DMF solution for various hours at $80^{\circ} \mathrm{C}$ to allow the PBASE molecules to adsorb by $\pi-\pi$ interactions ${ }^{23}$. After drying, another graphene film (smaller in surface area) was directly stacked onto the centre of first bottom graphene films to avoid the direct contact of upper graphene with the underlying gold substrates.

Junction of mechanically cleaved graphene via dry transfer. The bottom monolayer graphene was directly scotch taped onto silicon wafer (pre-cleaned by oxygen plasma), as a result, this graphene layer is almost wrinkle free and have no polymer residues. After PBASE SAM formation, another fresh exfoliated graphene was transferred on top to form the junctions via dry transfer stage ${ }^{30-32}$. These devices were fabricated in Class 1000 cleanroom.

Electronic measurement via EGaln Tip. In a home-built set-up similar to that reported by Whitesides and Chiechi et al. ${ }^{19,20}$, eutectic metal alloy EGaIn (75.5 wt.\% gallium and 24.5 wt.\% indium, from Sigma-Aldrich) was used to record 
the $J(\mathrm{~V})$ characteristics at room temperature. The cone-shaped $\mathrm{Ga}_{2} \mathrm{O}_{3} / \mathrm{EGaIn}$ top electrodes were fabricated and lifted by a $10-\mu \mathrm{l}$ glass syringe (Hamilton, 1701RNR) with a metal needle (Hamilton, conical shape 26s). To isolate from vibrations that are dominant sources of the failure of the junctions, a series of vibration damping systems were employed, one of which critical components of the EGaIn-setup is the micromanipulator (Leica) with only $1.0 \mathrm{~nm}$ per hour drifting. For the contact with bottom electrode, a micro-positioner (Crest innovation, S-725PLM) was used to hold a gold probe tip to form direct electrical contact with bottom gold substrate. All the $I-V$ measurements were recorded using a Keithley 6485 picoammeter.

DFT simulation. All calculations were carried out using DFT-based Vienna $a b$ initio simulation package (VASP) with Perdew-Burke-Ernzerhof approximation for the exchange-correlation functional, and frozen-core all-electron projectoraugmented wave method ${ }^{37-39}$. The cut-off energy for the expansion of plane-wave basis was set to $400 \mathrm{eV}$. The graphene/molecular/graphene junctions were modelled by sandwiching the molecules in between two $7 \times 7 \times 1$ graphene supercells, and the related Brillouin zone was sampled by gamma-centred $3 \times 3 \times 1 \kappa$-point meshes. To minimize the interaction between neighbour graphene surfaces, a $15-\AA$ vacuum was applied normal to graphene surface. All structures were optimized until the force on each atom is smaller than $0.02 \mathrm{eV}^{-1}$, in which van der Waals effects were included using DFT-D2 method of Grimme ${ }^{40}$.

\section{References}

1. Kim, K. S. et al. Large-scale pattern growth of graphene films for stretchable transparent electrodes. Nature 457, 706-710 (2009).

2. Wang, Y., Tong, S. W., Xu, X. F., Özyilmaz, B. \& Loh, K. P. Interface engineering of layer-by-layer stacked graphene anodes for high-performance organic solar cells. Adv. Mater. 23, 1514-1518 (2011).

3. Li, X. et al. Large-area synthesis of high-quality and uniform graphene films on copper foils. Science 324, 1312-1314 (2009).

4. Bae, S. K. et al. Roll-to-roll production of 30-inch graphene films for transparent electrodes. Nat. Nanotech. 5, 574-578 (2010).

5. Han, T. H. et al. Extremely efficient flexible organic light-emitting diodes with modified graphene anode. Nat. Photon. 6, 105-110 (2012).

6. Wang, G. et al. A new approach for molecular electronic junctions with a multilayer graphene electrode. Adv. Mater. 23, 755-760 (2011).

7. Liu, Y. et al. 'Quasi-freestanding' graphene-on-single walled carbon nanotube electrode for applications in organic light-emitting diode. Small 10, 944-949 (2014).

8. Xu, Y., Bai, H., Lu, G., Li, C. \& Shi, G. Flexible graphene films via the filtration of water-soluble noncovalent functionalized graphene sheets. J. Am. Chem. Soc. 130, 5856-5857 (2008).

9. Inokuchi, H. Semi-and photo-conductivity of molecular single crystals. Anthracene and pyrene. Bull. Chem. Soc. Jpn 29, 131-133 (1956).

10. Jeon, N. J. et al. Efficient inorganic-organic hybrid perovskite solar cells based on pyrene arylamine derivatives as hole-transporting materials. J. Am. Chem. Soc. 135, 19087-19090 (2013).

11. Krishnan, K. S. \& Ganguli, N. Large anisotropy of the electrical conductivity of graphite. Nature 144, 667-667 (1939).

12. Schedin, F. et al. Detection of individual gas molecules adsorbed on graphene. Nat. Mater. 6, 652-655 (2007).

13. Dan, Y., Lu, Y., Kybert, N. J., Luo, Z. \& Johnson, A. C. Intrinsic response of graphene vapor sensors. Nano Lett. 9, 1472-1475 (2009).

14. Song, H. S. et al. Origin of the relatively low transport mobility of graphene grown through chemical vapor deposition. Sci. Rep. 2, 337 (2012).

15. Liang, X. et al. Toward clean and crackless transfer of graphene. ACS Nano 5, 9144-9153 (2011).

16. Huang, P. Y. et al. Grains and grain boundaries in single-layer graphene atomic patchwork quilts. Nature 469, 389-392 (2011).

17. Petrone, N. et al. Chemical vapor deposition-derived graphene with electrical performance of exfoliated graphene. Nano Lett. 12, 2751-2756 (2012).

18. Ivasenko, O. \& Perepichka, D. F. Mastering fundamentals of supramolecular design with carboxylic acids. Common lessons from X-ray crystallography and scanning tunneling microscopy. Chem. Soc. Rev. 40, 191-206 (2011).

19. Nerngchamnong, N. et al. The role of van der Waals interaction in the performance of molecular diodes. Nat. Nanotech. 8, 113-118 (2013).

20. Chiechi, R. C., Weiss, E. A., Dickey, M. D. \& Whitesides, G. M. Eutectic gallium-indium (EGaIn): A moldable liquid metal for electrical characterization of self-assembled monolayers. Angew. Chem. Int. Ed. 47, 142-146 (2008)

21. Simeone, F. C. et al. Defining the value of injection current and effective electrical contact area for EGaIn-based molecular tunneling junctions. J. Am. Chem. Soc. 135, 18131-18144 (2013).
22. Wan, A. et al. Reversible soft top-contacts to yield molecular junctions with precise and reproducible electrical characteristics. Adv. Funct. Mater. 24, 4442-4456 (2014).

23. Chen, R. J., Zhang, Y., Wang, D. \& Dai, H. Noncovalent sidewall functionalization of single-walled carbon nanotubes for protein immobilization. J. Am. Chem. Soc. 123, 3838-3839 (2001).

24. Lee, W. H. et al. Surface-directed molecular assembly of pentacene on monolayer graphene for high-performance organic transistors. J. Am. Chem. Soc. 133, 4447-4454 (2011).

25. Simmons, J. G. Generalized formula for the electric tunnel effect between similar electrodes separated by a thin insulating film. J. Appl. Phys. 34, 1793-1803 (2004).

26. Das, A. et al. Monitoring dopants by Raman scattering in an electrochemically top-gated graphene transistor. Nat. Nanotech. 3, 210-215 (2008).

27. Ferrari, A. C. et al. Raman spectrum of graphene and graphene layers. Phys. Rev. Lett. 97, 187401 (2006).

28. Dong, X. et al. Doping single-layer graphene with aromatic molecules. Small 5, 1422-1426 (2009).

29. Giovannetti, G. et al. Doping graphene with metal contacts. Phys. Rev. Lett. 101, 026803 (2008).

30. Dean, C. R. et al. Boron nitride substrates for high-quality graphene electronics. Nat. Nanotech. 5, 722-726 (2010).

31. Ponomarenko, L. A. et al. Tunable metal-insulator transition in double-layer graphene heterostructures. Nat. Phys. 7, 958-961 (2011).

32. Britnell, L. et al. Field-effect tunneling transistor based on vertical graphene heterostructures. Science 335, 947-950 (2012).

33. Britnell, L. et al. Electron tunneling through ultrathin boron nitride crystalline barriers. Nano Lett. 12, 1707-1710 (2012).

34. Xia, F., Perebeinos, V., Lin, Y. M., Wu, Y. \& Avouris, P. The origins and limits of metal-graphene junction resistance. Nat. Nanotech. 6, 179-184 (2011).

35. Ji, X., Zhang, J., Wang, Y., Qian, H. \& Yu, Z. A theoretical model for metal-graphene contact resistance using a DFT-NEGF method. Phys. Chem. Chem. Phys. 15, 17883-17886 (2013).

36. Gong, C. et al. First-principles study of metal-graphene interfaces. J. Appl. Phys. 108, 123711 (2010).

37. Kresse, G. \& Hafner, J. Ab initio molecular dynamics for liquid metals. Phys. Rev. B 47, 558-561 (1993).

38. Kresse, G. \& Hafner, J. Ab initio molecular dynamics for open-shell transition metals. Phys. Rev. B 48, 13115-13118 (1993).

39. Blöchl, P. E. Projector augmented-wave method. Phys. Rev. B 50, 17953-17979 (1994).

40. Grimme, S. Semiempirical GGA-type density functional constructed with a long-range dispersion correction. J. Comput. Chem. 27, 1787-1799 (2006).

\section{Acknowledgements}

K.P.L. thanks MOE Tier II grant 'Interface Engineering of Graphene Hybrids for Energy Conversion'. Grant Number: R-143-000-488-112. The Singapore National Research Foundation (NRF Award No. NRF-RF 2010-03 to C.A.N.) is kindly acknowledged for supporting this research. We thank J. WU and S.F. WANG for helpful discussions on FET device fabrication.

\section{Author contributions}

K.P.L. and C.A.N. supervised the project. Y.L. and L.Y. designed and performed the experiments. Y.P.L. prepared graphene samples and conducted the SAM formation. L.Y. performed the $J(\mathrm{~V})$ measurements. M.Y performed VASP DFT calculations. Y.Z., C.T.N. and N.N. helped to record and analyse the AFM, Raman, XPS, FTIR and UPS spectra. L.G. and L.L. helped to prepare graphene films and electrode fabrication. C.S.S.S. recorded the $I-V$ curves of exfoliated graphene samples. All authors contributed to writing the manuscript.

\section{Additional information}

Supplementary Information accompanies this paper at http://www.nature.com/ naturecommunications

Competing financial interests: The authors declare no competing financial interests.

Reprints and permission information is available online at http://npg.nature.com/ reprintsandpermissions/

How to cite this article: Liu, Y. et al. Giant enhancement in vertical conductivity of stacked CVD graphene sheets by self-assembled molecular layers. Nat. Commun. 5:5461 doi: 10.1038/ncomms6461 (2014). 